\title{
Vibroacoustic Independent Contributors and Active Control of Vibration and Sound in Double Walls: Part I. Vibroacoustic Modal Control*
}

\author{
Tsutomu KAIZUKA ${ }^{* *, * * *}$, Kimihiko NAKANO ${ }^{* *}$ and Nobuo TANAKA ${ }^{* * * *}$ \\ **Institute of Industrial Science, The University of Tokyo, \\ 4-6-1 Komaba, Meguro-ku, Tokyo, 153-8505 Japan \\ E-mail: tkaizuka@iis.u-tokyo.ac.jp; knakano@iis.u-tokyo.ac.jp \\ ***JSPS Research Fellow PD,

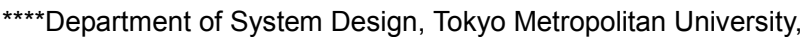 \\ 6-6 Asahigaoka, Hino-shi, Tokyo, 191-0065 Japan \\ E-mail: ntanaka@sd.tmu.ac.jp
}

\begin{abstract}
This paper is the first of two companion papers on active control based on two independent contributors to the structural kinetic and acoustic potential energy: vibroacoustic modes and clusters. An understanding of the vibroacoustic independent contributors clarifies the main causes of vibrations and sound, and facilitates a reasonable control system design, as reported in earlier literatures. In this paper, the theory of vibroacoustic modal control is applied to a double wall. The double wall consisted of a pair of panels, separated by an air gap and connected by mechanical springs, where the receiver panel is coupled with an acoustic enclosure. Velocity distributions on the panel's surface, which independently contribute to the sum of the structural kinetic energy of the panel and the acoustic potential energy in the enclosure, are derived. These velocity distributions are vibroacoustic modes. Filters are formulated to extract the individual vibroacoustic modes from the measured velocities. The validity of the developed theory is verified through numerical simulations.
\end{abstract}

Key words: Vibration, Sound, Noise, Double Wall, Active Control

\section{Introduction}

The magnitudes of structural vibration and sound are not necessarily proportional..$^{(1)-(8)}$ This vibroacoustic discrepancy creates a dilemma when trying to reduce vibration or sound levels. For example, Jones and Fuller ${ }^{(1)}$ applied an active control system to a simplified model of an aircraft fuselage to attenuate cabin noise. The control system consisted of microphones in the cabin and shakers attached to the fuselage. The microphones measured sound pressures in the cabin, and the shakers actuated the fuselage to minimize the measured sound pressures. This control method, which is based on the idea that sound radiated from vibrating structures can be reduced by controlling the structural vibrations generating the radiation, is called active structural acoustic control (ASAC). ASAC is a different noise control approach from active noise control (ANC), which is based on acoustic measurement and acoustic actuation. Because ASAC uses structural actuators, e.g. lowprofile piezoelectric patches mounted in the fuselage, it is much more compact than ANC, which uses acoustic actuators, e.g. bulky speaker and cabinet arrangements in the cabin. In this experiment, the cabin noise was successfully decreased; however, the fuselage 
vibration was increased. This approach, therefore, involved the risk that the increased vibration could cause the aircraft to fail.

Vibration and sound should be controlled in an integrated framework, and a control system that can handle both vibration and sound is desired. Such a control system can be designed reasonably and strategically with an understanding the independent contributors to the structural kinetic and acoustic potential energy. In this paper, these are referred to as vibroacoustic independent contributors. Understanding these contributors gives an insight into the dominant cause of vibration and sound. In this regard, however, conventional independent contributors, such as structural modes and acoustic modes, are not suitable, because these modes are not consistently orthogonal in structural-acoustic coupled systems. The structural modes are orthogonal to the structural kinetic energy but not to the acoustic potential energy, and the acoustic modes are orthogonal to the acoustic potential energy but not to the structural kinetic energy. To the best of the authors' knowledge, there are two approaches for deriving these contributors in terms of the structural vibrations: vibroacoustic modes; ${ }^{(9),(10)}$ and clusters of the structural modes in symmetric vibroacoustic systems. ${ }^{(8),(11),(12)}$ The emphasis of the derivation in the structural vibration domain is on a control system design based on structural sensors, e.g. piezoelectric films mounted in the aircraft fuselage, which is compact in comparison with acoustic sensors, such as microphones placed in the cabin.

Vibroacoustic modes are velocity distributions on the structural surface, which contribute independently to the sum of the structural kinetic energy and the acoustic potential energy in an acoustically enclosed space (acoustic power in an open space). Kaizuka and Tanaka,${ }^{(9)}$ and Kaizuka ${ }^{(10)}$ formulated distributed and point sensor-based spatial filters to estimate vibroacoustic modes from the measured velocities, for arbitrary vibroacoustic systems in an enclosed space as well as in an open space.

It is known that in symmetric vibroacoustic systems, uncoupled structural mode shapes are clustered according to an even or odd function, and these clusters are independent contributors to both the structural kinetic energy and the acoustic potential energy in an enclosed space (acoustic power in an open space). However, this independence is effective in units of the clusters but not in units of the structural modes included in each cluster. Clark and Fuller ${ }^{(13)}$ developed distributed sensor-based spatial filters to detect clusters associated with an even function from the measured vibrational data, for rectangular panels in an open space. Tanaka and Snyder ${ }^{(14)}$ developed point sensor-based spatial filters, which can measure each cluster related to an even or odd function, for rectangular panels in an open space. Kaizuka and Tanaka ${ }^{(15),(16)}$ generalized this work to arbitrary symmetric vibroacoustic systems in open and enclosed spaces.

This paper describes vibroacoustic modal control applied to double walls, and the accompanying paper ${ }^{(17)}$ describes cluster control applied to double walls. Double walls are often used to achieve high sound insulation with lightweight structures, e.g. aircraft fuselage shells and double windows. However, sound insulation performance generally deteriorates at low frequencies. Active control systems, such as ASAC and ANC, have been expected to provide a solution to this problem; ${ }^{(18)-(28)}$ however, the vibroacoustic discrepancy produces the dilemma of being able to reduce either the vibration or the sound, especially in the case of ASAC. The primary purpose of this paper is to control both the vibrations and sound in double walls by controlling the vibroacoustic modes. A secondary purpose is to compare the advantage and disadvantages of vibroacoustic modal control and cluster control through a case study of double walls. Cluster control is discussed in the accompanying paper.

Section 2 reviews the theory of double walls. Section 3 focuses on the theory of vibroacoustic modes in double walls. In Sec. 4, numerical simulations are conducted to verify the performance of vibroacoustic modal control. Section 5 summarizes the findings of this paper. 


\section{Theory: Double Walls}

A rectangular double wall like that illustrated in Fig. 1 is theoretically developed, following the approach of Cheng et al. ${ }^{(29)}$ The rectangular double wall is composed of two uniform flat panels, separated by an air gap and connected by $I$ mechanical springs. The upper panel is subjected to an external acoustic excitation, i.e. a disturbance input, and $J$ point forces, i.e. control inputs, whereas the lower panel is coupled to an enclosure. These two panels are flexible, and all other walls surrounding the air gap and enclosure are rigid.

If the vibrational behaviors of the upper and lower panels are described by the summation of $M_{1}$ and $M_{2}$ structural modes, respectively, the vibration velocities on these two panels, $v_{1}(\mathbf{r}, \omega)$ and $v_{2}(\mathbf{r}, \omega)$, are given by

$$
\begin{aligned}
& v_{1}(\mathbf{r}, \omega)=\boldsymbol{\varphi}_{1}{ }^{T}(\mathbf{r}) \mathbf{b}_{1}(\omega), \\
& v_{2}(\mathbf{r}, \omega)=\boldsymbol{\varphi}_{2}{ }^{T}(\mathbf{r}) \mathbf{b}_{2}(\omega),
\end{aligned}
$$

where $\mathbf{r}$ is the location, $\omega$ is the angular frequency, $\boldsymbol{\varphi}_{1}(\mathbf{r})$ and $\boldsymbol{\varphi}_{2}(\mathbf{r})$ are $M_{1}$ - and $M_{2}$-length column vectors consisting of the shapes of the uncoupled structural modes of the upper and lower panels, respectively, $\mathbf{b}_{1}(\omega)$ and $\mathbf{b}_{2}(\omega)$ are $M_{1}$ - and $M_{2}$-length column vectors consisting of the complex amplitudes of the structural modes of the upper and lower panels, respectively, and the superscript $T$ denotes the transpose.

Likewise, if the acoustical behaviors in the air gap and the enclosure are described by the summation of $N_{1}$ and $N_{2}$ acoustic modes, respectively, the acoustic pressures in these two cavities, $p_{1}(\mathbf{r}, \omega)$ and $p_{2}(\mathbf{r}, \omega)$, are given by

$$
\begin{aligned}
& p_{1}(\mathbf{r}, \omega)=\boldsymbol{\psi}_{1}{ }^{T}(\mathbf{r}) \mathbf{a}_{1}(\omega), \\
& p_{2}(\mathbf{r}, \omega)=\boldsymbol{\psi}_{2}{ }^{T}(\mathbf{r}) \mathbf{a}_{2}(\omega),
\end{aligned}
$$

where $\psi_{1}(\mathbf{r})$ and $\boldsymbol{\psi}_{2}(\mathbf{r})$ are $N_{1}$ - and $N_{2}$-length column vectors consisting of the uncoupled acoustic mode shapes of the air gap and the enclosure, respectively, and $\mathbf{a}_{1}(\omega)$ and $\mathbf{a}_{2}(\omega)$ are $N_{1}$ and $N_{2}$-length column vectors consisting of the complex amplitudes of the acoustic modes of the air gap and the enclosure, respectively.

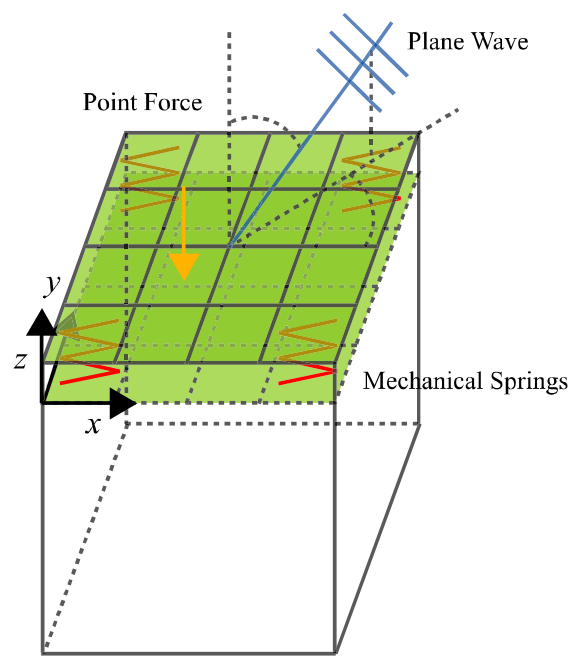

Fig. 1 A double wall with mechanical springs, which is subjected to an outer plane wave and a point force. 
Following the approach of Cheng et al., ${ }^{(29)}$ the complex amplitudes of the structural modes of the upper panel are extended to

$$
\begin{aligned}
& \mathbf{b}_{1}(\omega)=\mathbf{Y}_{1}(\omega)\left[\int_{S_{1}} \boldsymbol{\varphi}_{1}(\mathbf{r}) p(\mathbf{r}, \omega) d \mathbf{r}+\boldsymbol{\Phi}_{1, f} \mathbf{f}(\omega)\right. \\
& \left.-\mathbf{B}_{1,1}{ }^{T} \mathbf{a}_{1}(\omega)-\frac{1}{j \omega} \boldsymbol{\Phi}_{1, K} \mathbf{K}\left\{\boldsymbol{\Phi}_{1, K}{ }^{T} \mathbf{b}_{1}(\omega)-\boldsymbol{\Phi}_{2, K}{ }^{T} \mathbf{b}_{2}(\omega)\right\}\right],
\end{aligned}
$$

where $S_{1}$ is the surface area of the upper panel; $p(\mathbf{r}, \omega)$ is the outside acoustic pressure; $\boldsymbol{\Phi}_{1, f}$ is an $M_{1} \times J$ matrix, which is dependent on the upper panel structural mode shapes and the locations of the point forces; $\mathbf{f}(\omega)$ is a $J$-length column vector consisting of the point forces; $\boldsymbol{\Phi}_{1, K}$ is an $M_{1} \times I$ matrix, which is dependent on the upper panel structural mode shapes and the locations of the mechanical springs; $\boldsymbol{\Phi}_{2, K}$ is an $M_{2} \times I$ matrix, which is dependent on the lower panel structural mode shapes and the locations of the mechanical springs; and $\mathbf{K}$ is an $I \times I$ diagonal matrix consisting of stiffness coefficients of the mechanical springs. $\mathbf{Y}_{1}(\omega)$ is an $M_{1} \times M_{1}$ diagonal matrix, whose components are the resonant coefficients of the upper panel structural mode and defined as

$$
Y_{m_{1}}(\omega)=\frac{j \omega}{M_{m_{1}}\left(\omega_{m_{1}}{ }^{2}-\omega^{2}+j 2 \zeta_{m_{1}} \omega_{m_{1}} \omega\right)},
$$

where $M_{m_{1}}$ is the modal mass of the $m_{1}$ th structural mode of the upper panel, $\omega_{m_{1}}$ is the natural angular frequency, and $\zeta_{m_{1}}$ is the damping ratio. In addition, $\mathbf{B}_{1,1}$ is an $N_{1} \times M_{1}$ matrix consisting of coupling coefficients between the upper panel structural modes and the acoustic modes of the air gap, and is defined as

$$
\mathbf{B}_{1,1}=\int_{S_{1}} \boldsymbol{\psi}_{1}(\mathbf{r}) \boldsymbol{\varphi}_{1}(\mathbf{r}) d \mathbf{r}
$$

Equation (2.5) can be rewritten as

$$
\mathbf{b}_{1}(\omega)=\mathbf{Y}_{1}(\omega)\left[\int_{S_{1}} \boldsymbol{\varphi}_{1}(\mathbf{r}) p(\mathbf{r}, \omega) d \mathbf{r}+\boldsymbol{\Phi}_{1, f} \mathbf{f}(\omega)-\mathbf{B}_{1,1}{ }^{T} \mathbf{a}_{1}(\omega)-\mathbf{C}_{1,1}(\omega) \mathbf{b}_{1}(\omega)+\mathbf{C}_{1,2}(\omega) \mathbf{b}_{2}(\omega)\right]
$$

where

$$
\begin{aligned}
& \mathbf{C}_{1,1}(\omega)=\frac{1}{j \omega} \boldsymbol{\Phi}_{1, K} \mathbf{K} \boldsymbol{\Phi}_{1, K}{ }^{T}, \\
& \mathbf{C}_{1,2}(\omega)=\frac{1}{j \omega} \boldsymbol{\Phi}_{1, K} \mathbf{K} \boldsymbol{\Phi}_{2, K}{ }^{T} .
\end{aligned}
$$

The components in $\mathbf{C}_{1,1}(\omega)$ are regarded as feedback coefficients of the structural modes of the upper panel through the mechanical springs, and the components of $\mathbf{C}_{1,2}(\omega)$ are the coupling coefficients between the structural modes of the upper and lower panels via the springs. The complex amplitudes of the lower panel structural modes are extended to

$$
\mathbf{b}_{2}(\omega)=\mathbf{Y}_{2}(\omega)\left[\mathbf{B}_{1,2}{ }^{T} \mathbf{a}_{1}(\omega)-\mathbf{B}_{2,2}{ }^{T} \mathbf{a}_{2}(\omega)+\frac{\boldsymbol{\Phi}_{2, K} \mathbf{K}}{j \omega}\left\{\boldsymbol{\Phi}_{1, K}{ }^{T} \mathbf{b}_{1}(\omega)-\boldsymbol{\Phi}_{2, K}{ }^{T} \mathbf{b}_{2}(\omega)\right\}\right],(2.1
$$

where $\mathbf{Y}_{2}(\omega)$ is an $M_{2} \times M_{2}$ diagonal matrix, whose components are the resonant coefficients of the structural mode of the lower panel and defined as

$$
Y_{m_{2}}(\omega)=\frac{j \omega}{M_{m_{2}}\left(\omega_{m_{2}}{ }^{2}-\omega^{2}+j 2 \zeta_{m_{2}} \omega_{m_{2}} \omega\right)},
$$


where $M_{m_{2}}$ is the modal mass of the $m_{2}$ th structural mode of the lower panel, $\omega_{m_{2}}$ is the natural angular frequency, and $\zeta_{m_{2}}$ is the damping ratio. $\mathbf{B}_{1,2}$ is an $N_{1} \times M_{2}$ matrix consisting of the coupling coefficients between the lower panel structural modes and the acoustic modes of the air gap, and is defined as

$$
\mathbf{B}_{1,2}=\int_{S_{2}} \boldsymbol{\psi}_{1}(\mathbf{r}) \boldsymbol{\varphi}_{2}(\mathbf{r}) d \mathbf{r}
$$

where $S_{2}$ is the surface area of the lower panel. $\mathbf{B}_{2,2}$ is an $N_{2} \times M_{2}$ matrix consisting of the coupling coefficients between the lower panel structural modes and the acoustic modes of the enclosure, and is defined as

$$
\mathbf{B}_{2,2}=\int_{S_{2}} \boldsymbol{\psi}_{2}(\mathbf{r}) \boldsymbol{\varphi}_{2}(\mathbf{r}) d \mathbf{r} .
$$

Equation (2.11) can be rewritten as

$$
\mathbf{b}_{2}(\omega)=\mathbf{Y}_{2}(\omega)\left[\mathbf{B}_{1,2}{ }^{T} \mathbf{a}_{1}(\omega)-\mathbf{B}_{2,2}{ }^{T} \mathbf{a}_{2}(\omega)+\mathbf{C}_{1,2}{ }^{T}(\omega) \mathbf{b}_{1}(\omega)-\mathbf{C}_{2,2}(\omega) \mathbf{b}_{2}(\omega)\right],
$$

where

$$
\mathbf{C}_{2,2}(\omega)=\frac{1}{j \omega} \boldsymbol{\Phi}_{2, K} \mathbf{K} \boldsymbol{\Phi}_{2, K}{ }^{T}
$$

Components of $\mathbf{C}_{2,2}(\omega)$ are interpreted as feedback coefficients of the lower panel structural modes through the mechanical springs.

The complex amplitudes of the acoustic modes of the air gap are extended to

$$
\mathbf{a}_{1}(\omega)=\mathbf{Z}_{1}(\omega)\left\{\mathbf{B}_{1,1} \mathbf{b}_{1}(\omega)-\mathbf{B}_{1,2} \mathbf{b}_{2}(\omega)\right\}
$$

where $\mathbf{Z}_{1}(\omega)$ is an $N_{1} \times N_{1}$ diagonal matrix, whose component are the resonant coefficients of the acoustic mode of the air gap and defined as

$$
Z_{n_{1}}(\omega)=\frac{j \omega \rho_{0} c_{0}^{2}}{\Lambda_{n_{1}}\left(\omega_{n_{1}}{ }^{2}-\omega^{2}+j 2 \zeta_{n_{1}} \omega_{n_{1}} \omega\right)}
$$

where $\rho_{0}$ is the air density, $c_{0}$ is the speed of sound in the air, $\Lambda_{n_{1}}$ is the modal volume of the $n_{1}$ th acoustic mode of the air gap, $\omega_{n_{1}}$ is the natural angular frequency, and $\zeta_{n_{1}}$ is the damping ratio. The complex amplitudes of the acoustic modes of the enclosure are extended to

$$
\mathbf{a}_{2}(\omega)=\mathbf{Z}_{2}(\omega) \mathbf{B}_{2,2} \mathbf{b}_{2}(\omega)
$$

where $\mathbf{Z}_{2}(\omega)$ is an $N_{2} \times N_{2}$ diagonal matrix, whose components are the resonant coefficients of the acoustic mode of the enclosure and defined as

$$
Z_{n_{2}}(\omega)=\frac{j \omega \rho_{0} c_{0}^{2}}{\Lambda_{n_{2}}\left(\omega_{n_{2}}{ }^{2}-\omega^{2}+j 2 \zeta_{n_{2}} \omega_{n_{2}} \omega\right)},
$$

where $\Lambda_{n_{2}}$ is the modal volume of the $n_{2}$ th acoustic mode of the enclosure, $\omega_{n_{2}}$ is the natural angular frequency, and $\zeta_{n_{2}}$ is the damping ratio.

Combination of Eqs. (2.8), (2.15), (2.17), and (2.19), provides the fully coupled structural acoustic responses of the double wall.

\section{Theory: Vibroacoustic Modes of Double Walls}

Derivation of the vibroacoustic modes begins with a description of the error criterion, 
i.e. the sum of the structural kinetic energies of the upper and lower panels and the acoustic potential energy in the enclosure. If the surfaces of the upper and lower panels are divided into $L_{1}$ and $L_{2}$ elements, respectively, the representative velocities of the elements of the two panels are given by

$$
\begin{aligned}
& \mathbf{v}_{1}(\omega)=\boldsymbol{\Phi}_{1}{ }^{T} \mathbf{b}_{1}(\omega), \\
& \mathbf{v}_{2}(\omega)=\boldsymbol{\Phi}_{2}{ }^{T} \mathbf{b}_{2}(\omega),
\end{aligned}
$$

where $\mathbf{v}_{1}(\omega)$ and $\mathbf{v}_{2}(\omega)$ are $L_{1}$ - and $L_{2}$-length column vectors consisting of the representative velocities of the elements of the upper and lower panels, respectively, $\boldsymbol{\Phi}_{1}$ is an $M_{1} \times L_{1}$ matrix, which is dependent on the shapes of the structural modes of the upper panel and the representative locations of the elements of the upper panel, and $\boldsymbol{\Phi}_{2}$ is an $M_{2} \times L_{2}$ matrix, which is dependent on the lower panel structural mode shapes and the representative locations of the lower panel elements. The structural kinetic energy of the upper and lower panels, $\operatorname{SKE}_{1}(\omega)$ and $\operatorname{SKE}_{2}(\omega)$, is given by

$$
\begin{aligned}
& \operatorname{SKE}_{1}(\omega)=\mathbf{v}_{1}{ }^{H}(\omega) \mathbf{M}_{1} \mathbf{v}_{1}(\omega), \\
& \operatorname{SKE}_{2}(\omega)=\mathbf{v}_{2}{ }^{H}(\omega) \mathbf{M}_{2} \mathbf{v}_{2}(\omega),
\end{aligned}
$$

where the superscript $H$ is the conjugate transpose, and $\mathbf{M}_{1}$ and $\mathbf{M}_{2}$ are the $L_{1} \times L_{1}$ and $L_{2} \times L_{2}$ diagonal matrices defined as, respectively,

$$
\begin{aligned}
& \mathbf{M}_{1}=\frac{\rho_{1} h_{1} \Delta S_{1}}{4} \mathbf{E}_{1}, \\
& \mathbf{M}_{2}=\frac{\rho_{2} h_{2} \Delta S_{2}}{4} \mathbf{E}_{2},
\end{aligned}
$$

where $\rho_{1}$ and $\rho_{2}$ are the densities of the upper and lower panels, $h_{1}$ and $h_{2}$ are the thicknesses of the upper and lower panels, $\Delta S_{1}$ and $\Delta S_{2}$ are the areas of the elements of the upper and lower panels, and $\mathbf{E}_{1}$ and $\mathbf{E}_{2}$ are $L_{1} \times L_{1}$ and $L_{2} \times L_{2}$ identity matrices, respectively. The acoustic potential energy in the enclosure, $A P E_{2}(\omega)$, is formulated as

$$
A P E_{2}(\omega)=\mathbf{v}_{2}{ }^{H}(\omega) \mathbf{R}_{2}(\omega) \mathbf{v}_{2}(\omega),
$$

where $\mathbf{R}_{2}(\omega)$ is an $L_{2} \times L_{2}$ symmetric matrix consisting of the acoustic transfer functions in the enclosure, and is defined as

$$
\mathbf{R}_{2}(\omega)=\frac{\Delta S_{2}^{2}}{4 \rho_{0} c_{0}{ }^{2}} \boldsymbol{\Psi}_{2}^{T} \mathbf{Z}_{2}^{*}(\omega) \boldsymbol{\Lambda}_{2} \mathbf{Z}_{2}(\omega) \boldsymbol{\Psi}_{2},
$$

where the superscript $*$ denotes the conjugate, $\boldsymbol{\Lambda}_{2}$ is an $N_{2} \times N_{2}$ diagonal matrix consisting of the modal volumes of the acoustic modes of the enclosure, and $\boldsymbol{\Psi}_{2}$ is an $N_{2} \times L_{2}$ matrix, which is dependent on the shapes of the acoustic modes of the enclosure and the adjoining locations to the representative points of the elements of the lower panel. Note that $\mathbf{R}_{2}(\omega)$ is positive definite, since the radiated acoustic potential energy is always positive for any non-zero vibration velocities. From Eqs. (3.3), (3.4), and (3.7), the error criterion is written as

$$
\begin{aligned}
& J(\omega)=\alpha(\omega)\left\{S K E_{1}(\omega)+S K E_{2}(\omega)\right\}+A P E_{2}(\omega) \\
& =\left[\begin{array}{l}
\mathbf{v}_{1}(\omega) \\
\mathbf{v}_{2}(\omega)
\end{array}\right]\left\{\alpha(\omega)\left[\begin{array}{cc}
\mathbf{M}_{1} & \mathbf{0} \\
\mathbf{0} & \mathbf{M}_{2}
\end{array}\right]+\left[\begin{array}{cc}
\mathbf{0} & \mathbf{0} \\
\mathbf{0} & \mathbf{R}_{2}(\omega)
\end{array}\right]\right\}\left[\begin{array}{l}
\mathbf{v}_{1}(\omega) \\
\mathbf{v}_{2}(\omega)
\end{array}\right],
\end{aligned}
$$

where $\alpha(\omega)$ is the positive real weighting factor required to keep a balance between the 
structural kinetic energy and the acoustic potential energy. The magnitude of the structural kinetic energy is usually much larger than that of the acoustic potential energy; thus, some weighting factor is required to equate these two physical quantities. A proper weighting factor will be presented at the end of this section. It is clear from Eq. (3.9) that the terms of $\mathbf{v}_{1}(\omega)$ and $\mathbf{v}_{2}(\omega)$ are independent contributors to $\alpha(\omega) S K E_{1}(\omega)$ and $\alpha(\omega) S K E_{2}(\omega)$, respectively, because $\alpha(\omega) \mathbf{M}_{1}$ and $\alpha(\omega) \mathbf{M}_{2}$ are diagonal, whereas the terms of $\mathbf{v}_{2}(\omega)$ are not independent contributors to $A P E_{2}(\omega)$, because $\mathbf{R}_{2}(\omega)$ is not diagonal. From the perspective of estimating the error criterion, $\alpha(\omega) S K E_{1}(\omega)$ and $\alpha(\omega) S K E_{2}(\omega)$ can be estimated from $\mathbf{v}_{1}(\omega)$ and $\mathbf{v}_{2}(\omega)$, by using $\alpha(\omega) \mathbf{M}_{1}$ and $\alpha(\omega) \mathbf{M}_{2}$, respectively; $A P E_{2}(\omega)$ can be estimated from $\mathbf{v}_{2}(\omega)$ using $\mathbf{R}_{2}(\omega)$. $\alpha(\omega) \mathbf{M}_{1}, \alpha(\omega) \mathbf{M}_{2}$, and $\mathbf{R}_{2}(\omega)$ are the frequency dependent filters for estimating the error criterion, i.e. the sum of $\alpha(\omega) \operatorname{SKE}_{1}(\omega), \alpha(\omega) \operatorname{SKE}_{2}(\omega)$, and $A P E_{2}(\omega)$, from the measured velocities, $\mathbf{v}_{1}(\omega)$ and $\mathbf{v}_{2}(\omega)$. The sizes of filters $\alpha(\omega) \mathbf{M}_{1}$ and $\alpha(\omega) \mathbf{M}_{2}$ are $L_{1}$ and $L_{2}$, respectively, as these matrices are diagonal, whereas the size of filter $\mathbf{R}_{2}(\omega)$ is $L_{2} \times L_{2}$, as this matrix is non-diagonal.

Derivation of the vibroacoustic modes proceeds with diagonalization of the set of matrices in braces in Eq. (3.9). First, consider the equation,

$$
\begin{aligned}
& {\left[\begin{array}{cc}
\mathbf{E}_{1} & \mathbf{0} \\
\mathbf{0} & \mathbf{Q}_{2}(\omega)
\end{array}\right]^{T}\left\{\alpha(\omega)\left[\begin{array}{cc}
\mathbf{M}_{1} & \mathbf{0} \\
\mathbf{0} & \mathbf{M}_{2}
\end{array}\right]+\left[\begin{array}{cc}
\mathbf{0} & \mathbf{0} \\
\mathbf{0} & \mathbf{R}_{2}(\omega)
\end{array}\right]\right\}\left[\begin{array}{cc}
\mathbf{E}_{1} & \mathbf{0} \\
\mathbf{0} & \mathbf{Q}_{2}(\omega)
\end{array}\right],} \\
& =\left[\begin{array}{cc}
\alpha(\omega) \mathbf{M}_{1} \\
\mathbf{0} & \alpha(\omega) \mathbf{Q}_{2}{ }^{T}(\omega) \mathbf{M}_{2} \mathbf{Q}_{2}(\omega)+\mathbf{Q}_{2}{ }^{T}(\omega) \mathbf{R}_{2}(\omega) \mathbf{Q}_{2}(\omega)
\end{array}\right]
\end{aligned}
$$

where $\mathbf{Q}_{2}(\omega)$ is an $L_{2} \times L_{2}$ matrix. Since $\mathbf{R}_{2}(\omega)$ is real and symmetric as described in Eq. (3.8), it has real eigenvectors. If $\mathbf{Q}_{2}(\omega)$ is a real orthogonal matrix whose columns are the eigenvectors of $\mathbf{R}_{2}(\omega)$, the following equation is satisfied,

$$
\mathbf{Q}_{2}^{T}(\omega) \mathbf{R}_{2}(\omega) \mathbf{Q}_{2}(\omega)=\mathbf{\Omega}_{2}(\omega),
$$

where $\boldsymbol{\Omega}_{2}(\omega)$ is an $L_{2} \times L_{2}$ diagonal matrix consisting of the eigenvalues of $\mathbf{R}_{2}(\omega)$. All the eigenvalues are positive, because $\mathbf{R}_{2}(\omega)$ is positive definite, as mentioned above. From the orthogonality of $\mathbf{Q}_{2}(\omega)$, the following equation is effective,

$$
\mathbf{Q}_{2}^{T}(\omega) \mathbf{Q}_{2}(\omega)=\mathbf{E}_{2} .
$$

Cazzolato and Hansen ${ }^{(30)}$ solved eigenvalue problems on the acoustic transfer function matrix in the enclosure, and found that the eigenvalues depend on the frequency, with peaks corresponding to the natural frequencies of the uncoupled acoustic modes, and the eigenvectors are substantially independent of the frequency, as these are identical to the acoustic mode shapes. Substituting Eqs. (3.11) and (3.12) into Eq. (3.10) yields

$$
\begin{aligned}
& {\left[\begin{array}{cc}
\alpha(\omega) \mathbf{M}_{1} & \mathbf{0} \\
\mathbf{0} & \alpha(\omega) \mathbf{Q}_{2}{ }^{T} \mathbf{M}_{2} \mathbf{Q}_{2}+\mathbf{Q}_{2}{ }^{T} \mathbf{R}_{2}(\omega) \mathbf{Q}_{2}
\end{array}\right] .} \\
& =\left[\begin{array}{cc}
\alpha(\omega) \mathbf{M}_{1} & \mathbf{0} \\
\mathbf{0} & \alpha(\omega) \mathbf{M}_{2}+\mathbf{\Omega}_{2}(\omega)
\end{array}\right]
\end{aligned}
$$

Substituting Eq. (3.13) into Eq. (3.9) leads to

$$
\begin{aligned}
& J(\omega)=\alpha(\omega)\left\{S K E_{1}(\omega)+S K E_{2}(\omega)\right\}+A P E_{2}(\omega) \\
& =\left[\begin{array}{c}
\mathbf{v}_{1}(\omega) \\
\mathbf{w}_{2}(\omega)
\end{array}\right]^{H}\left[\begin{array}{cc}
\alpha(\omega) \mathbf{M}_{1} & \mathbf{0} \\
\mathbf{0} & \alpha(\omega) \mathbf{M}_{2}+\boldsymbol{\Omega}_{2}(\omega)
\end{array}\right]\left[\begin{array}{c}
\mathbf{v}_{1}(\omega) \\
\mathbf{w}_{2}(\omega)
\end{array}\right],
\end{aligned}
$$


where $\mathbf{w}_{2}(\omega)$ is an $L_{2}$-length column vector, which is defined as

$$
\mathbf{w}_{2}(\omega)=\mathbf{Q}_{2}{ }^{T} \mathbf{v}_{2}(\omega) \text {. }
$$

It is clear from Eq. (3.14) that the terms of $\mathbf{v}_{1}(\omega)$ are independent contributors to $\alpha(\omega) \operatorname{SKE}_{1}(\omega)$, as mentioned previously, and the terms of $\mathbf{w}_{2}(\omega)$ are independent contributors to $\alpha(\omega) \operatorname{SKE}_{2}(\omega)+A P E_{2}(\omega)$, because $\alpha(\omega) \mathbf{M}_{2}+\boldsymbol{\Omega}_{2}(\omega)$ is diagonal. The terms of $\mathbf{w}_{2}(\omega)$ are referred to as the complex amplitudes of the vibroacoustic modes. The diagonal terms of $\alpha(\omega) \mathbf{M}_{2}+\boldsymbol{\Omega}_{2}(\omega)$ are regarded as relative contributions of the vibroacoustic modes to $\alpha(\omega) S K E_{2}(\omega)+A P E_{2}(\omega)$ if the complex amplitudes of these modes are equal, and the columns of $\mathbf{Q}_{2}$ are interpreted as shapes of the vibroacoustic modes. Moreover, considering Eq. (3.14), a weighting factor to equate the structural kinetic energy with the acoustic potential energy is formulated as

$$
\alpha(\omega)=\frac{\operatorname{rank}\left[\mathbf{M}_{1}(\omega)\right]+\operatorname{rank}\left[\mathbf{M}_{2}(\omega)\right]}{\operatorname{trace}\left[\mathbf{M}_{1}(\omega)\right]+\operatorname{trace}\left[\mathbf{M}_{2}(\omega)\right]} \times \frac{\operatorname{trace}\left[\mathbf{\Omega}_{2}(\omega)\right]}{\operatorname{rank}\left[\mathbf{\Omega}_{2}(\omega)\right]} .
$$

A comparison of Eqs. (3.9) and (3.14) shows that the matrix used to estimate $\alpha(\omega) S K E_{2}(\omega)+A P E_{2}(\omega)$ from $\mathbf{v}_{2}(\omega)$ is converted from a frequency dependent $L_{2} \times L_{2}$ non-diagonal matrix, $\alpha(\omega) \mathbf{M}_{2}+\mathbf{R}_{2}(\omega)$, to a combination of a frequency dependent $L_{2} \times L_{2}$ diagonal matrix, $\alpha(\omega) \mathbf{M}_{2}+\boldsymbol{\Omega}_{2}(\omega)$, and a frequency independent $L_{2} \times L_{2}$ nondiagonal matrix, $\mathbf{Q}_{2}$. This conversion reduces the complexity of the control system. Furthermore, the vibroacoustic modes can be truncated from $L_{2}$ to $L_{2}^{\prime}\left(<L_{2}\right)$, in reference to the relative contributions of these modes to $\alpha(\omega) S K E_{2}(\omega)+A P E_{2}(\omega)$, i.e. $\alpha(\omega) \mathbf{M}_{2}+\boldsymbol{\Omega}_{2}(\omega) . \alpha(\omega) \mathbf{M}_{2}+\boldsymbol{\Omega}_{2}(\omega)$ is then reduced to an $L_{2}^{\prime} \times L_{2}^{\prime}$ diagonal matrix, and $\mathbf{Q}_{2}$ is reduced to an $L_{2} \times L_{2}{ }^{\prime}$ nondiagonal matrix.

\section{Numerical Simulation: Vibroacoustic Modal Control of a Double Wall}

\subsection{Specification of a double wall}

The rectangular double wall analyzed in this section is illustrated in Fig. 1. This double wall is composed of two simply supported aluminum panels with a density of $2720 \mathrm{~kg} / \mathrm{m}^{3}$, Young's modulus of $71 \mathrm{GPa}$, and Poisson's ratio of 0.33 . The $x$-directional length of the panels is $0.70 \mathrm{~m}$ and the $y$-directional length is $0.55 \mathrm{~m}$. The upper panel is $0.0035 \mathrm{~m}$ thick, and the lower panel is $0.0040 \mathrm{~m}$ thick. The structural modes to be considered are $9 \times 7$ for the upper panel and $8 \times 6$ for the lower panel, and the modal damping ratios of these structural modes are set equal to 0.004 . The natural frequencies of the uncoupled structural modes of the upper panel are listed in Table 1, and those of the lower panel are listed in Table 2, where the frequency range of interest is below $400 \mathrm{~Hz}$. The density of air is taken as $1.21 \mathrm{~kg} / \mathrm{m}^{3}$, and the speed of sound in air as $340 \mathrm{~m} / \mathrm{s}$. The $z$-directional length of the air gap and the enclosure are 0.10 and $0.60 \mathrm{~m}$, respectively. The acoustic modes under consideration are $7 \times 6 \times 1$ for the air gap and $7 \times 6 \times 6$ for the enclosure, and the modal damping ratios of these acoustic modes are set equal to 0.004 . The natural frequencies of the uncoupled acoustic modes of the air gap are listed in Table 3, and those of the enclosure are listed in Table 4. Four mechanical springs are located at symmetric coordinates, $(1 / 8 \times 0.70 \mathrm{~m}, 1 / 8 \times 0.55 \mathrm{~m}),(7 / 8 \times 0.70 \mathrm{~m}, 1 / 8 \times 0.55 \mathrm{~m}),(7 / 8 \times 0.70 \mathrm{~m}, 7 / 8 \times 0.55$ $\mathrm{m})$, and $(1 / 8 \times 0.70 \mathrm{~m}, 7 / 8 \times 0.55 \mathrm{~m})$. The stiffness coefficients of these springs are assumed to be equal, where the amplitude of the stiffness is set in reference to the ratio between the stiffness of the mechanical springs and the aerostatic stiffness of the air gap, ${ }^{(29)}$

$$
\lambda=\frac{4 K_{s}}{K_{a}},
$$

where $K_{s}$ is the stiffness of each spring, and $K_{a}$ is the aerostatic stiffness defined as 


$$
K_{a}=\frac{\rho c_{0}{ }^{2} B_{1,1}{ }^{2}}{V_{1}},
$$

where $B_{1,1}$ is the coupling coefficient between the structural mode $(1,1)$ of the upper panel and the acoustic mode $(0,0,0)$ of the air gap, and $V_{1}$ is the volume of the air gap. When $\lambda=1$, i.e. $K_{s}=2.2 \times 10^{4} \mathrm{~N} / \mathrm{m}$ in this specific model, the energy is transmitted between the upper and lower panels through both the mechanical springs and the air gap with approximately equal dependence. ${ }^{(29)}$ Setting stiffness at this value makes it possible to observe the effect of the mechanical springs as well as the air gap on the coupling behavior between the two panels. In addition, it is assumed that each panel is divided equally into $4 \times 4$ elements for calculating the structural kinetic energy and the acoustic potential energy, and for deriving the vibroacoustic modes. External acoustic excitation is assumed to be an oblique plane wave with a pressure of $1 \mathrm{~Pa}$, an incident elevation angle of $\pi / 4$, and an incident azimuth angle of $\pi / 4$. Once these numerical values are specified, fully coupled vibroacoustic responses of the double wall can be calculated in accordance with the formulae presented in Sec. 3.

Table 1 Structural modes of the upper panel.

\begin{tabular}{|cc|}
\hline Mode & Natural Frequency $\mathrm{Hz}$ \\
\hline \hline$(1,1)$ & 45.9 \\
$(2,1)$ & 98.5 \\
$(1,2)$ & 131.1 \\
$(2,2)$ & 183.7 \\
$(3,1)$ & 186.2 \\
$(3,2)$ & 271.4 \\
$(1,3)$ & 273.1 \\
$(4,1)$ & 308.9 \\
$(2,3)$ & 325.7 \\
$(4,2)$ & 394.1 \\
\hline
\end{tabular}

Table 2 Structural modes of the lower panel.

\begin{tabular}{|cc|}
\hline Mode & Natural Frequency $\mathrm{Hz}$ \\
\hline \hline$(1,1)$ & 52.5 \\
$(2,1)$ & 112.6 \\
$(1,2)$ & 149.8 \\
$(2,2)$ & 209.9 \\
$(3,1)$ & 212.8 \\
$(3,2)$ & 310.1 \\
$(1,3)$ & 312.1 \\
$(4,1)$ & 353.0 \\
$(2,3)$ & 372.2 \\
\hline
\end{tabular}

Table 3 Acoustic modes of the air gap.

\begin{tabular}{|cc|}
\hline Mode & Natural Frequency $\quad \mathrm{Hz}$ \\
\hline \hline$(0,0,0)$ & 0 \\
$(1,0,0)$ & 242.9 \\
$(0,1,0)$ & 309.1 \\
$(1,1,0)$ & 393.1 \\
\hline
\end{tabular}

Table 4 Acoustic modes of the enclosure.

\begin{tabular}{|cc|}
\hline Mode & Natural Frequency $\mathrm{Hz}$ \\
\hline \hline$(0,0,0)$ & 0 \\
$(1,0,0)$ & 242.9 \\
$(0,0,1)$ & 283.3 \\
$(0,1,0)$ & 309.1 \\
$(1,0,1)$ & 373.2 \\
$(1,1,0)$ & 393.1 \\
\hline
\end{tabular}




\subsection{Vibroacoustic modal control of a double wall}

Feedforward control based on the vibroacoustic modes is applied to the double wall developed in the previous section. In principle, 16 vibroacoustic modes are derived for the lower panel, because the panel is divided into 16 elements. Prior to control, the fundamental properties of the vibroacoustic modes are determined through simulations.

Figure 2 shows the frequency characteristics of the relative contributions of the vibroacoustic modes to $\alpha(\omega) S K E_{2}(\omega)+A P E_{2}(\omega)$ if the complex amplitudes of these modes are equivalent, i.e. $\alpha(\omega) \mathbf{M}_{2}+\boldsymbol{\Omega}_{2}(\omega)$. The figure and Table 4 show that the contributions of the vibroacoustic modes are frequency dependent, with peaks corresponding to the natural frequencies of the acoustic modes of the enclosure. This observed behavior should be expected, considering that $\boldsymbol{\Omega}_{2}(\omega)$ is frequency dependent, with peaks relating to the natural frequencies of the acoustic modes of the enclosure, as mentioned in Sec. 3 and $\alpha(\omega)$ defined in Eq. (3.16). The figure also shows that four vibroacoustic modes contribute significantly, and the other modes contribute less, and their contributions converge at an equal value. Hence, in this specific case, $\alpha(\omega) S K E_{2}(\omega)+A P E_{2}(\omega)$ can be approximated by the four most significant vibroacoustic modes.

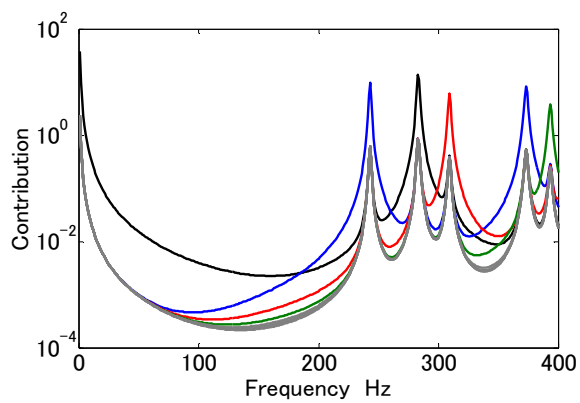

Fig. 2 Contributions of all 16 vibroacoustic modes to $\alpha(\omega) \operatorname{SKE}_{2}(\omega)+A P E_{2}(\omega)$, i.e. $\alpha(\omega) \mathbf{M}_{2}+\boldsymbol{\Omega}_{2}(\omega)$. The black line represents the first mode; the blue line, the second mode; the red line, the third mode; the green line, the fourth mode; and the gray lines, the fifth through 16th modes.

Figure 3 shows the shapes of the four vibroacoustic modes that contribute the most, i.e. the associated columns of $\mathbf{Q}_{2}(\omega)$, at an arbitrary chosen frequency $100 \mathrm{~Hz}$. The figure shows that the shapes of the vibroacoustic modes are identical to those of the acoustic modes of the enclosure, as mentioned in Sec. 3. Additional simulations confirmed that the shapes of the vibroacoustic modes are the same at other frequencies. Therefore, it is concluded that the shapes of the vibroacoustic modes are practically frequency independent.

Finally, feedforward control is carried out to minimize the error criterion defined in Eq. (3.14). It is assumed that the representative velocities of all the elements, i.e. 16 velocities for each panel, are measured. The measured 16 velocities on the upper panel, $\mathbf{v}_{1}(\omega)$, are input into the filters, $\alpha(\omega) \mathbf{M}_{1}$, which is a frequency dependent $16 \times 16$ diagonal matrix, and $\alpha(\omega) \operatorname{SKE}_{1}(\omega)$ is estimated. The 16 velocities measured on the lower panel, $\mathbf{v}_{2}(\omega)$, are input into the filters, $\mathbf{Q}_{2}(\omega)$, which is a $16 \times 4$ nondiagonal matrix and fixed at 100 $\mathrm{Hz}$, and the complex amplitudes of the four vibroacoustic modes that contribute the most, $\mathbf{w}_{2}(\omega)$, are calculated in accordance with Eq. (3.15). Note that $\alpha(\omega) S K E_{2}(\omega)+A P E_{2}(\omega)$ can be approximated from these four vibroacoustic modes, as determined in Fig. 2. The size of $\mathbf{Q}_{2}(\omega)$ is then reduced from $16 \times 16$ to $16 \times 4$. $\mathbf{Q}_{2}(\omega)$ is practically frequency independent, as confirmed in Fig. 3. Subsequently, the calculated four complex amplitudes are input into the filters, $\alpha(\omega) \mathbf{M}_{2}+\boldsymbol{\Omega}_{2}(\omega)$, which is a frequency dependent $4 \times 4$ diagonal matrix, and $\alpha(\omega) S K E_{2}(\omega)+A P E_{2}(\omega)$ is approximately obtained. If the vibroacoustic modes are not used, the error criterion is estimated in accordance with Eq. (3.9). The 16 velocities on the lower panel must then be 
input into the filters, $\alpha(\omega) \mathbf{M}_{2}+\mathbf{R}_{2}(\omega)$, which is a frequency dependent $16 \times 16$ non-diagonal matrix, to estimate $\alpha(\omega) S K E_{2}(\omega)+A P E_{2}(\omega)$. Knowledge of the vibroacoustic modes enables a significant simplification of the control system. A point force is assumed to be located at $(3 / 8 \times 0.70 \mathrm{~m}, 3 / 8 \times 0.55 \mathrm{~m})$. The amplitude and phase of the point force required to minimize the estimated error criterion are derived by conventional linear quadratic optimization. According to Secs. 2 and 3, the error criterion defined in Eq. (3.14) can be rewritten as

$$
J(\omega)=\mathbf{f}^{H}(\omega) \mathbf{A}(\omega) \mathbf{f}(\omega)+\mathbf{f}^{H}(\omega) \mathbf{b}(\omega)+\mathbf{b}^{H}(\omega) \mathbf{f}(\omega)+c(\omega),
$$

where

$$
\begin{aligned}
& \mathbf{A}(\omega)=\boldsymbol{\Phi}_{1, f}{ }^{T} \mathbf{D}(\omega) \boldsymbol{\Phi}_{1, f}, \\
& \mathbf{b}(\omega)=\boldsymbol{\Phi}_{1, f}{ }^{T} \mathbf{D}(\omega) \int_{S_{1}} \boldsymbol{\varphi}_{1}(\mathbf{r}) p(\mathbf{r}, \omega) d \mathbf{r}, \\
& c(\omega)=\int_{S_{1}} \boldsymbol{\varphi}_{1}{ }^{T}(\mathbf{r}) p^{*}(\mathbf{r}, \omega) d \mathbf{r D}(\omega) \int_{S_{1}} \boldsymbol{\varphi}_{1}(\mathbf{r}) p(\mathbf{r}, \omega) d \mathbf{r}, \\
& \mathbf{D}(\omega)=\alpha(\omega) \mathbf{G}_{1}{ }^{H}(\omega) \mathbf{\Phi}_{1} \mathbf{M}_{1} \mathbf{\Phi}_{1}{ }^{T} \mathbf{G}_{1}(\omega) \\
& +\mathbf{G}_{2}{ }^{H}(\omega) \boldsymbol{\Phi}_{2} \mathbf{Q}_{2}\left\{\alpha(\omega) \mathbf{M}_{2}+\mathbf{\Omega}_{2}(\omega)\right\} \mathbf{Q}_{2}{ }^{T} \mathbf{\Phi}_{2}{ }^{T} \mathbf{G}_{2}(\omega) \\
& \mathbf{G}_{1}(\omega)=\left[\mathbf{E}_{1}+\right. \\
& \left.\mathbf{Y}_{1}(\omega)\left\{\mathbf{B}_{1,1}{ }^{T} \mathbf{Z}_{1}(\omega) \mathbf{B}_{1,1}+\mathbf{C}_{1,1}(\omega)-\left(\mathbf{B}_{1,1}{ }^{T} \mathbf{Z}_{1}(\omega) \mathbf{B}_{1,2}+\mathbf{C}_{1,2}(\omega)\right) \mathbf{H}(\omega)\right\}\right]^{-1} \mathbf{Y}_{1}(\omega) \\
& \mathbf{G}_{2}(\omega)=\mathbf{H}(\omega)\left[\mathbf{E}_{1}+\right. \\
& \left.\mathbf{Y}_{1}(\omega)\left\{\mathbf{B}_{1,1}{ }^{T} \mathbf{Z}_{1}(\omega) \mathbf{B}_{1,1}+\mathbf{C}_{1,1}(\omega)-\left(\mathbf{B}_{1,1}{ }^{T} \mathbf{Z}_{1}(\omega) \mathbf{B}_{1,2}+\mathbf{C}_{1,2}(\omega)\right) \mathbf{H}(\omega)\right\}\right]^{-1} \mathbf{Y}_{1}(\omega) \\
& \mathbf{H}(\omega)=\left[\mathbf{E}_{2}+\mathbf{Y}_{2}(\omega)\left\{\mathbf{B}_{1,2}{ }^{T} \mathbf{Z}_{1}(\omega) \mathbf{B}_{1,2}+\mathbf{B}_{2,2}{ }^{T} \mathbf{Z}_{2}(\omega) \mathbf{B}_{2,2}+\mathbf{C}_{2,2}(\omega)\right\}\right]^{-1} \\
& \mathbf{Y}_{2}(\omega)\left\{\mathbf{B}_{1,2}{ }^{T} \mathbf{Z}_{1}(\omega) \mathbf{B}_{1,1}+\mathbf{C}_{1,2}{ }^{T}(\omega)\right\}
\end{aligned}
$$

Here, $\mathbf{E}_{1}$ and $\mathbf{E}_{2}$ are $M_{1} \times M_{1}$ and $M_{2} \times M_{2}$ identity matrices, respectively. Expressed in this form, the optimum control forces, which produce global minimum value of the quadratic function in Eq. (4.3), are determined by the relationship ${ }^{(31)}$

$$
\mathbf{f}(\omega)=-\mathbf{A}(\omega)^{-1} \mathbf{b}(\omega) \text {. }
$$

In addition to the weighting factor, $\alpha(\omega)$, which was defined in Eq. (3.16) and used in the simulations in Figs. 2 and 3, two other patterns of the weighting factors were also tried, for comparison. One pattern is a weighting factor 100 times the one defined in Eq. (3.16), where the emphasis is on controlling the panel vibration; the other is the weighting factor 0.01 times the one defined in Eq. (3.16), where the emphasis is on controlling sound in the enclosure. 


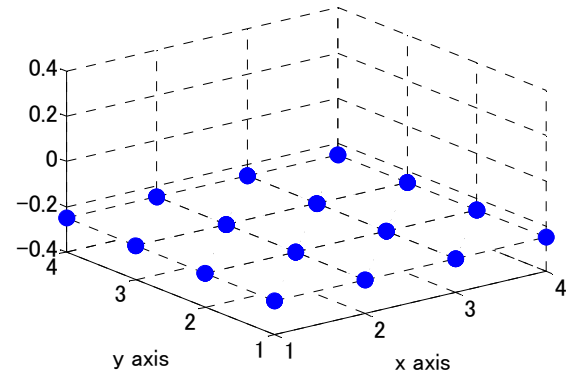

(a) First mode

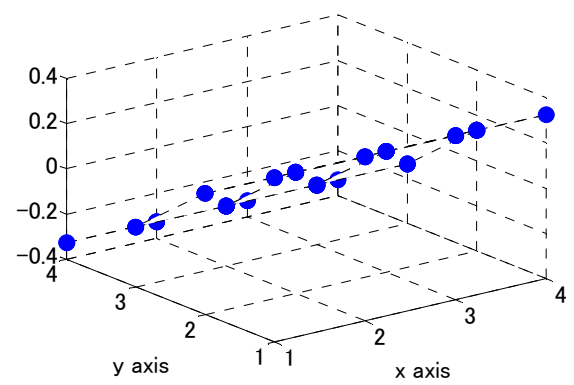

(c) Third mode

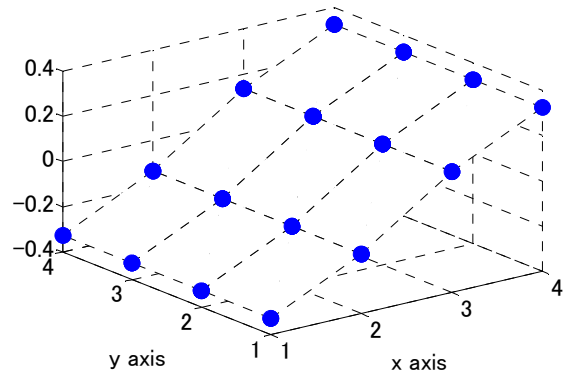

(b) Second mode

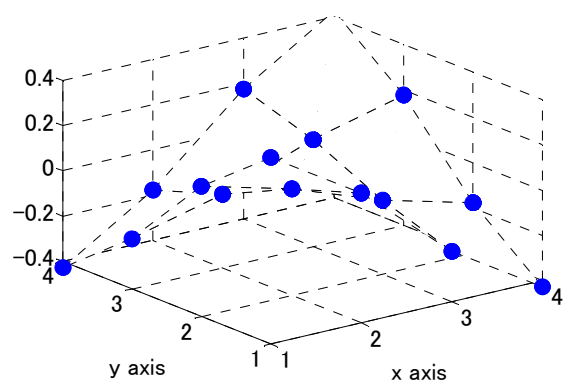

(d) Fourth mode

Fig. 3 Shapes of four most contributing vibroacoustic modes at $100 \mathrm{~Hz}$.

Figure 4 shows frequency characteristics of the double wall before and after vibroacoustic modal control. The figure shows that the vibroacoustic modal control with emphasis on the panel vibration works the best in the upper panel and the worst in the lower panel and the enclosure. The poor performance in the lower panel is because the magnitude of the structural kinetic energy of the upper panel is much larger than of the lower panel. In this context, the control system, which is designed to minimize the sum of the structural kinetic energies of the upper and lower panels, preferentially reduces the structural kinetic energy of the upper panel. The poor performance in the enclosure is not surprising. The figure shows that the vibroacoustic modal control with emphasis on sound in the enclosure works the best in the enclosure and the lower panel, and the worst in the upper panel. The vibroacoustic modal control with emphasis on both the panel vibration and sound in the enclosure, which is the key objective of this paper, provides the optimized performance for both the panels and the enclosure.

\section{Conclusions}

We investigated rectangular double walls consisting of a pair of uniform flat panels, which are separated by an air gap and connected with mechanical springs, where the receiver panel is coupled with an enclosure. In general, the sound insulation performance of double walls degrades at low frequencies. Although active control systems such as ASAC can improve the poor sound reduction performance in an enclosure, their use can increase the panel vibration. This vibroacoustic discrepancy may be reasonably solved by control strategies using error criteria components that individually contribute to both the structural kinetic energy of the panels and the acoustic potential energy in the enclosure. This is the principle underlying this work. 


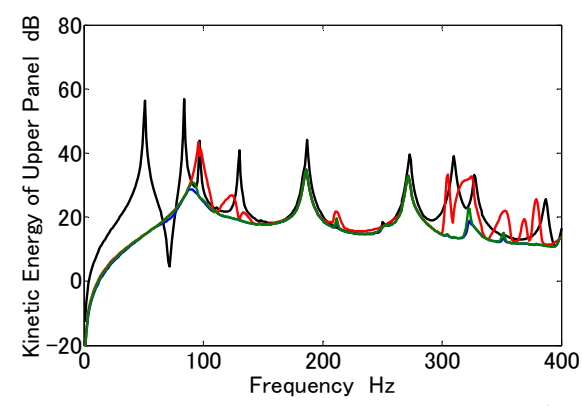

(a) Upper panel kinetic energy $\left(\mathrm{dB}\right.$ ref. $\left.=10^{-9} \mathrm{~J}\right)$.

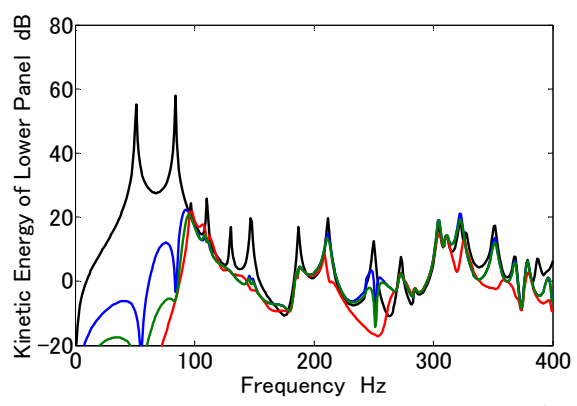

(b) Lower panel kinetic energy $\left(\mathrm{dB}\right.$ ref. $\left.=10^{-9} \mathrm{~J}\right)$.

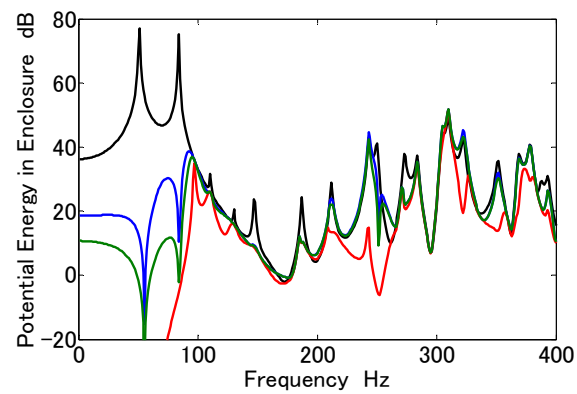

(c) Potential energy in the enclosure $\left(\mathrm{dB}\right.$ ref. $\left.=10^{-12} \mathrm{~J}\right)$.

Fig. 4 Frequency characteristics of a double wall before and after vibroacoustic modal control. The black line represents values before control; the blue line, values after control with emphasis on vibration; the red line, values after control with emphasis on sound; and the green line, values after control with emphasis on both vibration and sound.

When the mass of the panel and the acoustic transfer functions in the enclosure are given, velocity distributions, which independently contribute to both the structural kinetic energy of the panel and the acoustic potential energy in the enclosure, termed vibroacoustic modes, are available. Simulations demonstrated that the vibroacoustic mode shapes are identical to those of the uncoupled acoustic modes of the enclosure and are frequency independent. Therefore, the complex amplitude of each vibroacoustic mode can be calculated from measured velocities of the panel using a frequency independent spatial filter. Simulation also confirmed that the relative contributions of the vibroacoustic modes to the sum of the structural kinetic energy and the acoustic potential energy are frequency dependent with peaks related to the natural frequencies of the acoustic modes of the enclosure. The sum of the structural and acoustic energies can be estimated by inputting the calculated complex amplitudes of the vibroacoustic modes into the frequency dependent filters to provide added weights associated with the relative contributions of these modes. Order reduction can be achieved in reference to the relative contributions. If the panel velocities are represented by $L \times L$ elements and $L^{\prime}(<L)$ vibroacoustic modes contribute, then a frequency independent $L \times L^{\prime}$ nondiagonal matrix is required to calculate the complex amplitudes of the vibroacoustic modes, and a frequency dependent $L^{\prime} \times L^{\prime}$ diagonal matrix is needed to provide added weights associated with the relative contributions of these modes to the structural and acoustic energies. In contrast, if the vibroacoustic modes are not used, the acoustic potential energy of the enclosure must be estimated from the measured panel velocities by a frequency dependent $L \times L$ matrix consisting of acoustic transfer functions. Feedforward control with the error criteria being the vibroacoustic modes that contribute was simulated, and the panel vibration and the sound in the enclosure were successfully controlled.

Vibroacoustic modal control exactly reduces the sum of the structural kinetic energy and the acoustic potential energy. Hence, it is guaranteed that the sound transmission loss of double wall is improved while suppressing an increase in structural vibrations of the wall. 
However, the implementation of vibroacoustic modal control is not necessarily easy. Although independence of the vibroacoustic modes makes the control system simpler, the filters to estimate the relative contribution of these modes include the frequency dependence, which is still complicated. In other words, these filters are implemented in digital filters, which cause degree of computational burden. In the case where this calculation cost is allowable, vibroacoustic modal control is a good solution.

The second part of this work ${ }^{(17)}$ discusses another kind of vibroacoustic independent contributor, a cluster of structural modes in symmetric double walls. An advantage of this approach is that filters to extract each cluster are frequency independent. Therefore, these filters are composed of fixed gains. One of the disadvantages of cluster control is that this control method is not applicable to asymmetric double walls. Another disadvantage is that the independence of the clusters is effective in units of the clusters but not in units of the modes in each cluster. Thus, it is not strictly guaranteed in cluster control that sound is decreased while suppressing an increase in vibrations.

\section{Acknowledgement}

This work was financially supported by Grant-in-Aid for JSPS Fellows (No.18-7445).

\section{References}

(1) Jones, J.D. and Fuller, C.R., Active control of sound fields in elastic cylinders by multicontrol forces, AIAA Journal, Vol.27, No.7, (1989), pp.845-852.

(2) Fuller, C.R., Active control of sound transmission/radiation from elastic plates by vibration inputs, I. Analysis, Journal of Sound and Vibration, Vol.136, No.1, (1990), pp.1-15.

(3) Pan, J., Hansen, C.H., and Bies, D.A., Active control of noise transmission through a panel into a cavity: I. Analytical Study, Journal of the Acoustical Society of America, Vol.87, No.5, (1990), pp.2098-2108.

(4) Pan, J. and Hansen, C.H., Active control of noise transmission through a panel into a cavity: II. Experimental study, Journal of the Acoustical Society of America, Vol.90, No.3, (1991), pp.1488-1492.

(5) Snyder, S.D. and Hansen, C.H., Mechanisms of active noise control by vibration sources, Journal of Sound and Vibration, Vol.147, No.3, (1991), pp.519-525.

(6) Pan, J., Snyder, S.D., Hansen, C.H., and Fuller, C.R., Active control of far-field sound radiated by a rectangular panel: A general analysis, Journal of the Acoustical Society of America, Vol.91, No.4, (1992), pp.2056-2066.

(7) Snyder, S.D. and Hansen, C.H., Design of systems to control actively periodic sound transmission into enclosed spaces, Part I: Analytical models, Journal of Sound and Vibration, Vol.170, No.4, (1994), pp.433-449.

(8) Snyder, S.D. and Hansen, C.H., Design of systems to control actively periodic sound transmission into enclosed spaces, Part II: Mechanisms and trends, Journal of Sound and Vibration, Vol.170, No.4, (1994), pp.451-472.

(9) Kaizuka, T. and Tanaka, N., Vibroacoustic modes and active control of both vibration and sound, AIAA Journal, Vol.46, No.6, (2008), pp.1490-1504.

(10) Kaizuka, T., Vibroacoustic orthogonal contributors and active control of both structural vibration and sound, Ph.D. dissertation, Tokyo Metropolitan University, (2008).

(11) Cunefare, K.A., The minimum multimodal radiation efficiency of baffled finite beams, Journal of the Acoustical Society of America, Vol.90, No.5, (1991), pp.2521-2529.

(12) Currey, M.N. and Cunefare, K.A., The radiation modes of baffled finite plates, Journal of the Acoustical Society of America, Vol.98, No.3, (1995), pp.1570-1580.

(13) Clark, R.L. and Fuller, C.R., Modal sensing of efficient acoustic radiators with polyvinylidene fluoride distributed sensors in active structural acoustic control approaches, Journal of the Acoustical Society of America, Vol.91, No.6, (1992), pp.3321-3329. 
(14) Tanaka, N. and Snyder, S.D., Cluster control of a distributed-parameter planar structure: Middle authority control, Journal of the Acoustical Society of America, Vol.112, No.6, (2002), pp.2798-2807.

(15) Kaizuka, T. and Tanaka, N., Radiation clusters and the active control of sound transmission into a symmetric enclosure, Journal of the Acoustical Society of America, Vol.121, No.2, (2007), pp.922-937.

(16) Kaizuka, T. and Tanaka, N., Radiation clusters and the active control of sound transmission through symmetric structures into free space, Journal of Sound and Vibration, Vol.311, No.1-2, (2008), pp.160-183.

(17) Kaizuka, T., Nakano, K., and Tanaka, N., Vibroacoustic independent contributors and active control of vibration and sound in double walls: Part II. Cluster control, Journal of System Design and Dynamics, under review.

(18) Grosveld, F.W. and Shepherd, K.P., Active sound attenuation across a double wall structure, Journal of Aircraft, Vol.31, No.1, (1994), pp.223-227.

(19) Carneal, J.P. and Fuller, C.R., Active structural acoustic control of noise transmission through double panel systems, AIAA Journal, Vol.33, No.4, (1995), pp.618-623.

(20) Sas, P., Bao, C., Augusztinovicz, F., and Desmet, W., Active control of sound transmission through a double panel partition, Journal of Sound and Vibration, Vol.180, No.4, (1995), pp.609-625.

(21) Bao, C. and Pan, J., Experimental study of different approaches for active control of sound transmission through double walls, Journal of the Acoustical Society of America, Vol.102, No.3, (1997), pp.1664-1670.

(22) Pan, J. and Bao, C., Analytical study of different approaches for active control of sound transmission through double walls, Journal of the Acoustical Society of America, Vol.103, No.4, (1998), pp.1916-1922.

(23) Pan, X., Sutton, T.J., and Elliott, S.J., Active control of sound transmission through a double-leaf partition by volume velocity cancellation, Journal of the Acoustical Society of America, Vol.104, No.5, (1998), pp.2828-2835.

(24) Bao, C. and Pan, J., Active acoustic control of noise transmission through double walls: Effect of mechanical paths, Journal of Sound and Vibration, Vol.215, No.2, (1998), pp.395-398.

(25) Wang, C.-Y. and Vaicaitis, R., Active control of vibrations and noise of double wall cylindrical shells, Journal of Sound and Vibration, Vol.216, No.5, (1998), pp.865-888.

(26) Gardonio, P. and Elliott, S.J., Active control of structure-borne and airborne sound transmission through double panel, Journal of Aircraft, Vol.36, No.6, (1999), pp.1023-1032.

(27) Kaiser, O.E., Pietrzko, S.J., and Morari, M., Feedback control of sound transmission through a double glazed window, Journal of Sound and Vibration, Vol.263, No.4, (2003), pp.775-795.

(28) Li, Y.Y. and Cheng, L., Active noise control of mechanically linked double panel system coupled with an acoustic enclosures, Journal of Sound and Vibration, Vol.297, No.3-5, (2006), pp.1068-1074.

(29) Cheng, L., Li, Y.Y., and Gao, J.X., Energy transmission in a mechanically-linked double-wall structures coupled to an acoustic enclosure, Journal of the Acoustical Society of America, Vol.117, No.5, (2005), pp.2742-2751.

(30) Cazzolato, B.S. and Hansen, C.H., Active control of sound transmission using structural error sensing, Journal of the Acoustical Society of America, Vol.104, No.5, (1998), pp.2878-2889.

(31) Nelson, P.A. and Elliott, S.J., Active Control of Sound, Academic Press, (1992). 